\title{
Myasthenia Gravis in Patients with Histopathologically Diagnosed Thymoma at Sanglah General Hospital: A Case Series
}

\author{
I Gusti Ayu Sri Mahendra Dewi ${ }^{1 *}$, Desak Putu Gayatri Saraswati Seputra ${ }^{2}$ \\ ${ }^{1}$ Department of Anatomical Pathology, Faculty of Medicine, Udayana University -Sanglah Hospital, Denpasar, Indonesia \\ ${ }^{2}$ Ari Canti Hospital, Gianyar, Indonesia
}

\section{ARTICLE INFO}

Received : 01 August 2020

Reviewed : 15 September 2020

Accepted : 22 January 2021

\section{Keywords:}

co-occurrence, myasthenia gravis, thymoma

\author{
*Corresponding author: \\ I Gusti Ayu Sri Mahendra Dewi \\ Department of Anatomical Pathology, \\ Faculty of Medicine, Udayana \\ University - Sanglah Hospital, \\ Denpasar, Indonesia \\ mahendradewi@rocketmail.com
}

\begin{abstract}
Introduction: Thymoma is a neoplasm of the thymus gland which arises from the epithelial tissue. Thymoma is frequently related to various paraneoplastic syndromes, most commonly myasthenia gravis. Up to $50 \%$ of patients with thymoma are reported to develop myasthenia gravis, whereas $10-15 \%$ of patients with myasthenia gravis are found to have thymoma.
\end{abstract}

\begin{abstract}
Case Presentation: This case series reported 3 patients with myasthenia gravis which co-occurred with thymoma at Sanglah General Hospital from 2018 to 2019. The patients' ages range from 33 to 61 years (mean age $46 \pm 11$ years) with a 2:1 male-to-female ratio. The diagnosis of myasthenia gravis was made by the presentation of typical myasthenia gravis appearances, the fluctuating intensity of ocular and bulbar muscle weakness (all three cases), and limbs and axial muscle weakness (one case); no respiratory muscle involvement was found. Physical and supporting examinations including electroneuromyography results were coherent with myasthenia gravis. The diagnosis of thymoma was made by highly suggestive appearance in Thorax Multi Sliced Computed Tomography (MSCT) scan examination and histopathologic findings. MSCT scan examination showed a well-circumscribed ovoid solid mass in the mediastinum (left anterosuperior, superior, and anterior to middle right mediastinum) sized from $1.8 \times 1.5 \times 2.9 \mathrm{~cm}$ to $8.1 \times 9.9 \times 9.7 \mathrm{~cm}$, enhanced with contrast injection with areas of calcification and central necrotic ( 1 case) and no dissemination to regional lymph node and neighboring organs. All cases received acetylcholinesterase inhibitors and underwent extended thymectomy. Histopathological examination showed accordance with thymoma features of subtype $A, A B$, and $B 1$.
\end{abstract}

Conclusions: Clinicians should be aware of the thymoma and myasthenia gravis co-existence possibility to ensure early recognition and appropriate management.

\section{INTRODUCTION}

Thymoma is a neoplasm of the thymus gland which originates from the epithelial tissues. Thymoma is frequently associated with several paraneoplastic syndromes (PNS). Approximately, $50-70 \%$ of patients with thymoma come up with other presumable paraneoplastic etiology of the autoimmune condition [1]. Myasthenia gravis is found to be one of the most common paraneoplastic diseases in thymoma patients [2]. Myasthenia gravis is characterized by fluctuating weakness affecting the ocular, bulbar, extremity, and, in more severe cases, may also involve respiratory muscle which can be life-threatening [3]. Up to $50 \%$ of patients with thymoma are reported to develop myasthenia gravis $[1,3,4]$. Conversely, in myasthenia gravis diagnosed patients, the underlying abnormal histology features are oftentimes found in the thymic gland [5]. A total of $10-15 \%$ of patients with myasthenia gravis are found to have thymoma $[3,4]$.

Thymoma-associated myasthenia gravis (T-MG) is known to arise due to the ability of the epithelial neoplastic thymoma cells which are encircled by $T$ cells to express epitopes cross-reactive with acetylcholine receptor (AChR) and other skeletal muscle proteins like titin and ryanodine-receptor [4]. The immune response aimed at those epitopes triggers humoral autoimmunity and antibody production which simultaneously react in 
the same manner as neuromuscular junction and skeletal muscle components [3].

Considering the frequent co-occurrence of thymoma and myasthenia gravis, we report on three cases of myasthenia gravis in patients with histopathologically diagnosed thymoma at Sanglah General Hospital, a tertiary hospital in Denpasar, Bali in 2018-2019.

\section{CASE PRESENTATION}

We report three patients diagnosed with myasthenia gravis which occurred concurrently with thymoma at Sanglah General Hospital from 2018 to 2019. Clinical features, imaging, and histopathological findings were presented for each case. All cases were adults with the age range from 33 to 61 years with a mean age of 46 \pm 11 years. Out of three patients, 2 were male. The following clinical manifestations were found in the cases: one patient presented with ocular muscle weakness, blurred vision, and general weakness while two others experienced ocular muscle weakness, difficulty swallowing, and dysphonia, with no limb or axial muscle weakness. The clinical symptoms were fluctuating in intensity. All cases belong to subgroup IIB according to the Myasthenia Gravis Foundation of America (MGFA) clinical classification system. Physical and supporting examinations including an electroneuromyography test were performed. The results were coherent with myasthenia gravis, and diagnoses were made.
Thorax Multi Sliced Computed Tomography (MSCT) scan examination with and without contrast was performed in all cases. Well circumscribed ovoid solid mass in mediastinum was found in all three cases. Specific mass locations were found to be in the left anterosuperior, superior, and anterior to middle right mediastinum with the size ranging from $1.8 \times 1.5 \times 2.9$ $\mathrm{cm}$ to $8.1 \times 9.9 \times 9.7 \mathrm{~cm}$. All masses showed contrast enhancement. Calcification and central necrosis were found in one case. No spread to regional lymph nodes and dissemination to neighboring organs were noted (Figure 1).

All cases received acetylcholinesterase inhibitors and additionally underwent extended thymectomy as a measure of diagnosis establishment and treatment. The resection samples were sent to the Anatomical Pathology Laboratory, and the diagnosis was confirmed for each case through histopathological examination which showed accordance with thymoma features. Histopathological results showed subtypes $A, A B$, and $B 1$ of thymoma. Thymoma type $A$ in histopathological examination showed hyperplasia of spindle and ovalshaped cells with fine chromatin and inconspicuous nucleoli. Among these cells, there was a small distribution of immature lymphocytes. The cells were arranged in fascicular and lobulated patterns separated by fibrous connective tissue. The capsule of fibrous connective tissue lined the outer part of the tumor. Type $A B$ thymoma morphologically appeared to be a

Figure 1.

Overview of thorax Multi Sliced Computed Tomography (MSCT) examination in axial section. (A) Mediastinal mass with a size of $1.8 \times 1.5 \times 2.9 \mathrm{~cm}$; (B) Mediastinal mass with a size of $4.48 \times 7.42 \times 4.85 \mathrm{~cm}$.
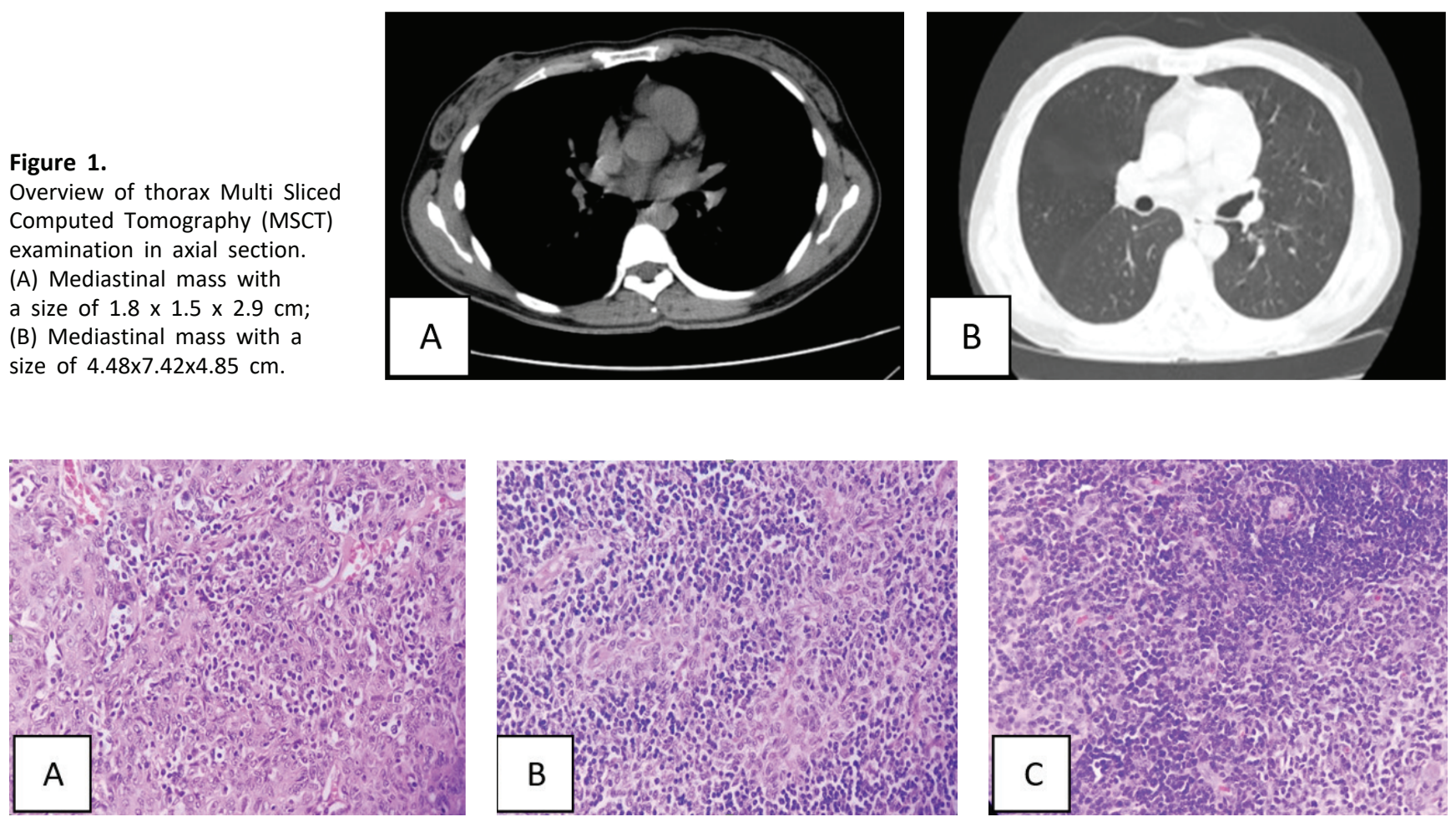

Figure 2. Histopathologic figures of thymoma (H\&E, 400x). (A) Thymoma type A; (B) Thymoma type AB; and (C) Thymoma type B1 
mixture of the spindle to oval-shaped cells with lymphocyte cells in varying numbers, whereas type B1 thymoma had similar morphology to the thymus (Figure 2).

\section{DISCUSSION}

Thymoma-associated myasthenia gravis (T-MG) is myasthenia gravis which occurs concurrently with thymoma. Thymoma is a neoplasm of the thymus gland which arises from the epithelial tissue [1]. Thymoma is frequently related to paraneoplastic syndrome (PNS). Myasthenia gravis is the most common PNS associated with thymoma, where up to $50 \%$ of thymoma patients develop myasthenia gravis $[1,3,4]$. On the contrary, 10 $15 \%$ of patients with myasthenia gravis are found to have a thymoma $[3,4]$.

T-MG is induced by the ability of epithelial neoplastic thymoma cells which are encircled by $T$ cells to express numerous self-like antigens including the acetylcholine receptor (AChR) and other skeletal muscle proteins like titin and ryanodine-receptor epitopes [4]. This phenomenon leads to humoral immune response and antibody production to epitopes on the neuromuscular junction and skeletal protein which resemble the epithelial neoplastic thymoma cells $[1,3,6]$.

T-MG has equal gender distribution. Our findings found that out of three patients reported, 2 were male. T-MG affects people of all ages with the peak age at onset of around 50 years [4]. Previous studies state that T-MG's mean age of onset is approximately 47 years [7], which shows an agreement with our case series findings.

The diagnosis of myasthenia gravis is made based on the typical clinical history and supporting examination. Additionally, thymoma should be demonstrated to establish a T-MG diagnosis [8]. The cardinal feature of myasthenia gravis is fluctuating muscle weakness which worsens with activities and improves with rest. Weakness may affect the ocular, bulbar, limb, and axial muscles, and less frequently involves the respiratory muscle. The most common symptom at its initial presentation is ocular muscle weakness, which occurs in $85 \%$ of cases. The involvement of bulbar muscle is visible in approximately $60 \%$ of patients. Ocular and bulbar weaknesses were found in all three cases reported. Bulbar muscle involvement in our case was presented as difficulty swallowing and loss of speech. Involvement of the limbs in myasthenia gravis is usually seen as predominantly proximal muscle weakness and was only found in one of our cases. Weakness involving respiratory muscles is rarely the presenting feature in the early onset disease [6]. No respiratory muscle involvement was reported in our patients. The fluctuating fatigability shown in our cases corresponds to the typical myasthenia gravis clinical hallmark. Physical and supporting examination results were also coherent with myasthenia gravis.

All cases belong to subgroup IIB according to the Myasthenia Gravis Foundation of America (MGFA) clinical classification system. Classification is made based on predominance in muscle group involvement and their severity. Class IIB comprises diseases with mild weaknesses predominantly manifested in oropharyngeal and/or respiratory muscles. They may also have lesser or equal involvement of limb and/or axial muscles and ocular muscle weaknesses of any severity [9]. This finding differs from a recent publication in Mexico which found class IIIB to be the most frequent subgroups in patients with T-MG (39\%) [10]. The discrepancy probably occurs due to differences in patient sociodemographic backgrounds.

The diagnosis of thymoma in our cases was made by the highly suggestive appearance in MSCT scan examination and histopathologic findings. Thymomas in MSCT scan appeared as a well-defined homogenous mass with round or lobulated shape and showed contrast enhancement. The tumor can be outlined by fat and may contain areas of calcification. Thymomas are usually discovered to be $5-10 \mathrm{~cm}$ in size at presentation, although these tumors have been reported to size from a few millimeters to $34 \mathrm{~cm}$ [11]. Thymomas on radiological findings of thorax MSCT scan obtained in this case series were found to be well-circumscribed ovoid solid masses, specifically in the left anterosuperior, superior, and anterior to the middle right mediastinum. The size ranged from $1.8 \times 1.5 \times 2.9 \mathrm{~cm}$ to $8.1 \times 9.9$ $x \quad 9.7 \mathrm{~cm}$. All masses showed contrast enhancement. Heterogenous contrast enhancement was seen in one case similarly in which calcification and central necrotic were found. Homogeneous enhance $\neg$ ment is a typical character of thymoma, even though heterogeneity can be observed in one-third of thymomas due to areas of necrosis, hemorrhage, or cystic changes $[11,12]$.

The definitive diagnosis of thymoma was finally established by histopathological examination postsurgery. Thymomas are classified by World Health Organization (WHO) into six separate histopathological subtypes namely types $A, A B, B 1, B 2, B 3$, and $C$. Thymoma originates from the epithelial cells and is associated with the proliferation of $\mathrm{T}$ lymphocyte in variable degree. WHO classifies each subtype according to the morphology of the epithelial cells and the number of associated T lymphocytes [1,12]. According to a recent study, type B1 thymoma is the most common histopathological subtype presenting with myasthenia gravis [13]. However, histopathological results in our case series showed no inclination to specific subtypes.

Surgery has been the mainstay of treatment for thymoma. Surgery assists in the histopathological evaluation and staging process and, in most cases, is 
used as the first-line treatment modality. It is advisable to perform immediate and complete surgical resections for resectable tumors $[1,6]$. The recommended surgical procedure is by extended thymectomy which completely removes the thymus and the surrounding mediastinal fat to assure no macroscopically invisible invasion remains [2]. Extended thymectomy procedures were performed in all reported cases. Post-operative radiation therapy and neoadjuvant or adjuvant chemotherapy may also be performed depending on the stage of thymoma and the resectability of the tumor $[1,12]$.

Radical excision of a thymoma mostly cures thymic neoplasia. However, patients will continue to suffer from myasthenia gravis following thymectomy. This underlines the need for sustained follow-up and pharmacologic therapy. First-line pharmacological therapy for myasthenia gravis is acetylcholinesterase inhibitors while immunosuppressive drugs may be used as the second choice or as an addition [8]. All cases received acetylcholinesterase inhibitors.

The prognosis of thymoma is tightly linked to two factors, their staging, and completeness of surgical resection [12]. There are still controversies regarding whether or not myasthenia gravis affects the prognosis of thymomas and vice versa $[1,6,8,14]$.

\section{CONCLUSIONS}

Thymoma is a neoplasm of the thymus gland which arises from the epithelial tissue. Thymoma is frequently related to various paraneoplastic syndromes, most commonly myasthenia gravis. Clinicians should be aware of this co-existence possibility to ensure early recognition and appropriate management.

\section{DECLARATIONS}

\section{Competing of Interest}

The authors declare no competing interest in this study.

\section{Acknowledgment}

The authors wish to thank the Head of Anatomical Pathology Laboratory Sanglah Hospital Denpasar and all staffs that contribute to the completion of this case series.

\section{REFERENCES}

1. Kumar R. Myasthenia gravis and thymic neoplasms: a brief review. World J Clin Cases. 2015;3(12):980-3.

2. Zhang Z, Cui $Y$, Jia $R$, et al.Myasthenia gravis in patients with thymoma affects survival rate following extended thymectomy. Oncol Lett. 2016;11:4177-82.

3. Romi F, Hong Y, Gilhus NE. Pathophysiology and immunological profile of myasthenia gravis and its subgroups. Curr Opin Immunol. 2017;49:9-13.

4. Beydoun SR, Gong $H$, Ashikian N, Rison RA. Myasthenia gravis associated with invasive malignant thymoma: two case reports and a review of the literature. J Med Case. 2014;8:340.

5. Panse RI, Bismuth J, Cizeron-Clairac G, et al. Thymic remodeling associated with hyperplasia in myasthenia gravis. J Autoimmun. 2010;43(5-6):401-12.

6. Trouth AJ, Dabi A, Solieman N, et al. Myasthenia gravis: a review. Autoimmune Dis. 2012:1-10.

7. Maggi L, Andreetta F, Antozzi C, et al. Thymomaassociated myasthenia gravis: outcome, clinical and pathological correlations in 197 patients on a 20-year experience. J Neuroimmunol. 2008;201-202:237-44.

8. Romi F. Thymoma in myasthenia gravis: from diagnosis to treatment. Autoimmune Dis. 2011:1-5.

9. Jaretzki A, Barohn RJ, Ernstoff RM, et al. Myasthenia gravis recommendations for clinical research standards. Neurology. 2000;55:16-23.

10. Cacho-Díaz B, Salmerón-Moreno K, LorenzanaMendoza NA, et al. Myasthenia gravis as a prognostic marker in patients with thymoma. J Thorac Dis. 2018;10(5):1-7.

11. Maron EM. Imaging thymoma. J Thorac Oncol. 2010;5:296-303.

12. Benveniste MF, Rosado-de-Christenson ML, Sabloff $\mathrm{BS}$, et al. Role of imaging in the diagnosis, staging, and treatment of thymoma. RadioGraphics. 2011;31:1847-61.

13. Agrawal M, Uppin MS, Uppin SG, et al. Thymoma diagnosis and categorization in the current scenario: morphological analysis based on interobserver variability. Ann Thorac Med. 2020;15:90-4.

14. Samuel O, David O, Toluwalase A, et al. Myasthenia gravis, thymoma, and thymectomy: the clinical interplay. Int J Clin Biomed Res. 2018;4(2):80-3. 